\title{
Author Index, Vol. 15, 1995
}

\section{$(\mathrm{A})=$ Abstract; Vol. 15, Supplement 1, has its own Author Index} Ager,A. 200(A) Alexander, K. 131 Ali,S.A. 210(A) Allegra,A. 283 Aloisi,C. 283 Altavilla,D. 80 Andreassen, A.K. 117 Ansell, G. 65 Arfors,K.E. 265 Ariwodola, J.O. 92 Arnold, F. 211 (A) Baatz, H. 85 Bannai, S. 206(A) Bardhan,K.D. 204(A) Barham,G.S. 208(A) Bari, F. 92 Bassani, C. 75 Baydoun,A.R. 205(A) Beard, R.W. 206 (A) Beattie, C.M.M. 208 (A) Becchetti, E. 181 Bell,F. 203(A) Benediktsson, K. 150(A) Berchiolli, R. 60 Bjørnerheim, R. 117 Björquist, P. 147(A) Black, CM. 202(A) Bodin,Ph. 1 Bolinder,J. 143(A) Bollinger,A. 10,53, 193,277 Borgström, P. 28,265 Bornmyr, S. 143(A) Bosnian, J. 271 Boström,S. 147(A) Boucher, N.R. 207(A) Bouskela,E. 170,293 Breit, G.A. 28 Brown, M.D. Ill Brown, N.J. 200 (A), 202 (A), 204 (A), 207 (A), 211(A) Brown, W. 203(A) Bruno, S. 75 Buemi,M. 283 Bull, H.A. 205 (A) Bull,R. 65 Burnstock, G. 1

Campo,G.M. 80 Canale,P. 80 Caputi,A.P. 80 Carvalho,D. 202(A) Caspary, L. 131

Catapano, G. 60 Chaloupka, K. 125 Chambers, A.F. 201 (A) Chaudhry,H. 210(A) Chen, C.-J. 21 Cherry, G. 211(A) Chmielewska, J. 145(A) Chong, C.-K. 21 Christ, F. 14 Cordiner, S. 209 (A) Corica,F. 283 Creutzig, A. 131 Crosignani, A. 75 Cugno, M. 75

Dattola, A. 283 Dell’Omo,G. 60 Denuzzo, G. 283 DeValle, G. 75 Ditano, G. 80 Donyo,K.A. 293 Dörffler-Melly, J. 10 Dormandy, J.A. 203(A),

210(A) Dowd,P.M. 205(A)

Egbrink, M.G.A. oude 200 (A),

271 Egginton, S. 209(A) Ellis, C.G. 223 Engelhardt, T. 43 Eriksson, B.I. 145(A) Eriksson, E. 145(A), 146(A), 149 (A)

Fahrig,C. 287 Felländer, G. 143(A) Ferrari, M. 60 Fevang, B.T. 325 Fevang, J. 325 Finn, A. 203(A) Fischer, M. 53 Foong, L.C. 206 (A) Franzeck, U.K. 10,53,193 Freni,F. 80 Friesenecker, B. 238 Froesch, E.R. 10 Furrer, J. 193

Gamble, J. 14, 206 (A), 208 (A) Garnham, A. 202 (A) Gartside, LB. 14,206 (A), 208 (A) Geigant,E. 150(A) Ghattaura, I.K. 210(A) Gislason, H. 325 Grattan,M. 210(A) Gray,W. 208(A) Gregory, A.K. 210(A) Grewal,P.S. 208(A) Grönroos, J. 144(A) Groom, A.C. 201(A), 223 Gullichsen, E. 144(A) Gustafsson, U. 144(A), 148(A), 163

Halvarsson, M. 145 (A) Harris, A.G. 85 Hart, I.R. 201(A) He,C.F. 211(A) Heidrich, H. 287 Heimann, A. 37 Hoffmann, U. 53,277 Holmdahl,L. 146(A) Höper,J. 186 Hothersall, J. 205 (A) Hsu, R. 250

Hudlicka, O. Ill,202 (A) Hunter, R.D. 209(A) Huseby,N. 155 Hussain, M.A. 10 Hutchinson, J. 209(A)

Iabichella, L. 60 Intaglietta, M. 28,217,238, 265 Ioculano, M. 80 Ishii,T. 206(A) Jacob, S. 204(A) Jahn,H. 186 Jian,Z. 143(A) Johnson, P.C. 217,218 Johnston, P.W. 208(A) 
Kalia,N. 204(A) Kaplan, V. 277 Kempski, O. 37 Kendall, S. 205 (A) Kernick, D.P. 203(A) Khokha, R. 201 (A) Kirkeby,O.J. 149(A), 316 Kjellström, B.T. 148(A) Koop, S. 201 (A) Kremer, E. 301 Kretschmar, K. 43

Krombach, F. 85 Kumar, P. 209(A) Kumar, S. 209(A) Kuttila,K. 144(A) Kvernebo, K. 117 Lamah,M. 203 (A), 210 (A) Länne,T. 145(A) Lee,J. 255

Levick,J.R. 65, 206 (A) Lewis, D.H. 144(A), 163 Lilli,C. 18Г Lin,B.J. 21 Linde,B. 143(A) Lindley,M. 209(A) Ljung,C. 145(A) Locci,P. 181 Loesch, A. 1 Lubbers, D.W. 131 Luostarinen, R. 149(A) Luzzi,K. 201(A)

McDonald, D.M. 208 (A) MacDonald, I.C. 201 (A) Mahy,I.R. 137 Makki,R.F. Ill Manciet, L.H. 231 Mann, G.E. 205 (A), 206 (A) Marasini, B. 75 Marinucci, L. 181 Marshall, J.F. 201(A) Marshall, J.M. 204(A) Martin, A. 203(A) Mason, R.M. 206 (A) Mathieu-Costello, O. 231 Mattsson, C. 145(A) Mawson,D.M. 210(A) May,M.J. 207(A) Mayhan,W.G. 48 Mazzoni, M.C. 265 Mehta,J. 149(A), 150(A) Melillo,E. 60 Messmer, K. 14,244 Michel, C.C. 205 (A), 208 (A) Milner,P. 1 Moffitt,H. 208(A) Mohan, J. 204(A) Morris, V.L. 201(A) Mortimer, P.S. 65, 203 (A), 210(A) Moser,C.M. 14 Myint,Y. 211(A) Myklebust,R. 155

331

Nelimarkka, O. 144(A) Nevalainen, T. 144(A) Niinikoski, J. 144(A) Niklas, M. 14 Nilsson,J. 146(A) Nolte,D. 244

Olsen, H. 145(A)

Pajulo,O. 147(A) Parbhoo, S.P. 125 Pearson, J.D. 202 (A), 206 (A),

207 (A) Pedrinelli, R. 60 Perbeck,L. 150(A) Peter, K. 14 Petroni, L. 75 Pfíster,G. 193 Philp, E.A. 209 (A) Pickelmann, S. 244 Pipia,C. 75 Pleschka, K. 92 Potter, R.F. 223 Price, F.M. 206(A)

Quarmby, S. 209 (A)

Rautanen, M. 144(A) Rawlins, M. 144(A) Rebuck,N. 203(A) Reed, M.W.R. 202(A), 204 (A), 207 (A), 209 (A),

211(A) Rees, R.C. 201 (A), 210(A) Refior,H.J. 14

Reikerås, O. 155 Reilly,C.S. 211(A) Reneman,R.S. 200(A), 271 Renvall,S. 147(A) Ribatti,D. 181 Richardson, V.J. 210(A) Risberg,B. 143(A), 145(A),

146(A), 149(A) Rise,I.R. 149(A), 316 Risöe, C. 316 Rochester, J.R. 202(A) Roman, N. 148(A) Roncali,L. 181 Rubanyi, G.M. 170 Rubinstein, I. 48

Saini,A. 201(A) Saitta,A. 80

Saldeen,T. 149 (A), 150 (A) Schmid-Schönbein, G.W. 255,

309 Schmidt, E.E. 201(A) Schmidt, J.A. 28 Secomb,T.W. 250 Seifalian, A.M. 125 Seiffge,D. 301 Seller, Z. 201(A) Serjeant, G.R. 204(A) Shore, A.C. 137, 210(A) Simonsen, S. 117 Siow, R.C.M. 206 (A) Sirsjö,A. 144(A) Sjöberg,F. 144(A), 163 Skalak, T.C. 265 Slaaf,D.W. 200 (A), 271

Smith, L.D.R. 137 Solders, G. 150(A) Sollevi,A. 144(A) Squadrito,F. 80,283 Squadrito, G. 80,283 Stanton, A.W.B. 65 Steinbauer, M. 85,244 Stephenson, T.J. 209(A) Steurer, J. 277 Strecker,U. 37 Sunesson, A. 148(A) Sutherland, LA. 206(A) Suzuki, H. 309 Svanes,K. 325 Svensson,B. 148(A) Sweiry,J.H. 206(A)

Tai,T.-Y. 21

Tangelder, G.J. 200 (A), 271 Taub,D.D. 210(A) Thomas, D.C. 206(A) Thorborg,P. 163 Thum,J. 131 Tooke,J.E. 137, 203 (A) Tsai,A.G. 238 Tseng, C.-H. 21 Turner, A.R. 210(A) Tyml,K. 231 Uçkay, I. 53 
Vaage,J. 146(A), 147 (A) Valen,G. 146 (A), 147 (A) Vermiglio, G. 283 Vetter,W. 277 Viljanto,J. 147(A) Voigt,B. 287

Wang,M. 209(A) Warnke,K,C. 265 Weiss, J.B. Ill Wellander,E. 149(A) Wen,S. 10,53

Wikström,T. 149(A) Wileman, S.M. 205 (A) Wiman,B. 146 (A), 147 (A) Winnerkvist, A. 146

(A),

147 (A) Wnuk,G. 287 Wrigley, S.J. 223

Xiu, R.-j. 143(A)

Yanar,A. 193 Ying,X.-y. 143(A) Youngs, S.J. 210(A) Ytrehus,K. 155

Zaugg-Vesti, B.R. 193 Zeintl,H. 244 Zeni,S. 75 Ziegler, C. von 193 Zweifach, B.W. 309

332

Author Index Vol. 15, 1995 